\title{
Picometer-scale surface roughness measurements inside hollow glass fibres
}

\author{
C. Brun, ${ }^{1,2}$ X. Buet,,${ }^{1,3}$ B. Bresson, ${ }^{4}$ M. S. Capelle, ${ }^{1,2}$ M. Ciccotti, ${ }^{4}$ A. \\ Ghomari, ${ }^{3}$ P. Lecomte, ${ }^{1,2}$ J. P. Roger, ${ }^{2}$ M. N. Petrovich,${ }^{5}$ F. Poletti, ${ }^{5}$ D. \\ J. Richardson, ${ }^{5}$ D. Vandembroucq, ${ }^{1, *}$ and G. Tessier ${ }^{2,3}$ \\ ${ }^{1}$ Laboratoire PMMH, ESPCI, CNRS UMR 7636, Univ. Paris 6 UPMC, Univ. Paris 7 Diderot, \\ 10 rue Vauquelin 75231 Paris cedex 05, France \\ 2 Institut Langevin, ESPCI, CNRS UMR 7587, 1 rue Jussieu, 75238 Paris cedex 05, France \\ ${ }^{3}$ Neurophotonics Laboratory, CNRS UMR 8250, Univ. Paris Descartes, Paris, France \\ ${ }^{4}$ Laboratoire PPMD-SIMM, ESPCI, CNRS UMR 7615, Univ. Paris 6 UPMC, \\ 10 rue Vauquelin 75231 Paris cedex 05, France \\ ${ }^{5}$ Optoelectronics Research Centre, Univ. Southampton, \\ Highfield, Southampton, SO17 1BJ, UK \\ *damienvdb@pmmh.espci.fr
}

\begin{abstract}
A differential profilometry technique is adapted to the problem of measuring the roughness of hollow glass fibres by use of immersion objectives and index-matching liquid. The technique can achieve picometer level sensitivity. Cross validation with AFM measurements is obtained through use of vitreous silica step calibration samples. Measurements on the inner surfaces of fibre-sized glass capillaries drawn from high purity suprasil F300 tubes show a sub-nanometer roughness, and the roughness power spectrum measured in the range $\left[5 \cdot 10^{-3} \mu \mathrm{m}^{-1}-10^{-1} \mu \mathrm{m}^{-1}\right]$ is consistent with the description of the glass surface as a superposition of frozen capillary waves. The surface roughness spectrum of two capillary tubes of differing compositions can be quantitatively distinguished.
\end{abstract}

(C) 2014 Optical Society of America

OCIS codes: (120.6660) Surface measurements, roughness; (120.4290) Nondestructive testing; (060.5295) Photonic crystal fibers; (240.5770) Roughness.

\section{References and links}

1. F. Poletti, M. N. Petrovich, and D. J. Richardson, "Hollow core photonic bandgap fibres; technology and applications," Nanophotonics 2, 315-340 (2013).

2. V. A. J. M. Sleiffer, J. Yongmin, N. K. Baddela, J. Surof, M. Kuschnerov, V. Veljanowski, J. R. Hayes, N. V. Wheeler, E. R. N. Fokoua, J. P. Wooler, D. R. Gray, N. H. L. Wong, F. R. Parmigiani, S. U. Alam, M. N. Petrovich, F. Poletti, D. J. Richardson, and H. D. Waardt, "High capacity mode-division multiplexed optical transmission in a novel 37-cell hollow-core photonic bandgap fiber," J. Lightwave Technol. 32, 854 (2014).

3. P. J. Roberts, F. Couny, H. Sabert, B. J. Mangan, D. P. Williams, L. Farr, M. W. Mason, A. Tomlinson, T. A. Birks, J. C. Knight, and P. S. Russell, "Ultimate low loss of hollow-core photonic crystal fibres," Opt. Express 13, 237 (2005).

4. E. N. Fokoua, F. Poletti, and D. J. Richardson, "Analysis of light scattering from surface roughness in hollow-core photonic bandgap fibers," Opt. Express 20, 20980 (2012).

5. N. V. Wheeler, M. N.Petrovich, R. Slavík, N. Baddela, E. J. R. Hayes, D. Gray, F. Poletti, and D. J. Richardson, "Wide-bandwidth, low-loss, 19-cell hollow core photonic band gap fiber and its potential for low latency data transmission," in "National Fiber Optic Engineers Conference," (OSA, 2012).

6. F. Poletti, N. V. Wheeler, N. Baddela, E. N. Fokoua, J. R. Hayes, R. Slavík, and D. J. Richardson, "Towards high-capacity fibre-optic communications at the speed of light in vacuum," Nat. Photon. 7, 279-284 (2013).

7. J. Jäckle and K. Kawazaki, "Intrinsic roughness of glass surfaces," J. Phys. Condens. Matter 7, 4351-4358 (1995).

\#223129 - \$15.00 USD Received 15 Sep 2014; revised 7 Nov 2014; accepted 10 Nov 2014; published 19 Nov 2014

(C) 2014 OSA 1 December $2014 \mid$ Vol. 22, No. 24 | DOI:10.1364/OE.22.029554| OPTICS EXPRESS 29554 
8. T. Sarlat, A. Lelarge, E. Søndergård, and D. Vandembroucq, "Frozen capillary waves on glass surfaces: an AFM study," Eur. Phys. J. B 54, 121-127 (2006).

9. M. C. Phan-Huy, J. M. Moison, J. A. Levenson, S. Richard, G. Mélin, M. Douay, and Y. Quiquempois, "Surface roughness and light scattering in a small effective area microstructured fiber," J. Lightwave Technol. 27, 15971604 (2009).

10. A. Tay, C. Thibierge, D. Fournier, C. Fretigny, F. Lequeux, C. Monteux, J. P. Roger, and L. Talini, "Probing thermal waves on the free surface of various media: Surface fluctuation specular reflection spectroscopy," Rev. Sci. Instrum. 79 (2008).

11. B. Pottier, G. Ducouret, C. Fretigny, F. Lequeux, and L. Talini, "High bandwidth linear viscoelastic properties of complex fluids from the measurement of their free surface fluctuations," Soft Matter 7, 7843-7850 (2011).

12. A. Raudsepp, C. Fretigny, F. Lequeux, and L. Talini, "Two beam surface fluctuation specular reflection spectroscopy," Rev. Sci. Instrum. 83 (2012).

13. P. Gleyzes and A. C. Boccara, "Interferometric polarization picometric profile .1. single detector approach," J. Opt. 25, 207-224 (1994).

14. http://www.bruker.com/products/surface-analysis/atomic-force-microscopy/dimension-icon/technical-details.html

15. D. Derks, D. G. A. L. Aarts, D. Bonn, H. N. W. Lekkerkerker, and A. Imhof, "Suppression of thermally excited capillary waves by shear flow," Phys. Rev. Lett. 97, 038301 (2006).

16. M. Thiebaud and T. Bickel, "Nonequilibrium fluctuations of an interface under shear," Phys. Rev. E 81, 031602 (2010).

\section{Introduction}

Hollow core-photonic bandgap fibres (HC-PBGFs), in which a periodic cladding made of air holes allows light to be guided in a central air core, are of great interest as a potential transmission medium in next-generation optical communication systems. Their nonlinearity and latency are much lower compared to classical solid-core [1,2], and they offer reduced Rayleigh scattering and absorption by impurities or phonons. However, losses in these fibres are still one order of magnitude higher than in typical single mode fibres. Scattering due to surface roughness at the numerous air/glass interfaces present in these structures, particularly those at the hollow core boundary, has been identified as a major loss mechanism [3]. In particular, components of roughness at short spatial frequencies (i.e. below $1 \mu \mathrm{m}^{-1}$ ) seem to have the highest impact on losses [4]. Longitudinally, roughness should therefore be characterized over distances of up to several hundreds of micrometers.

The optimization of multi-mode propagation offers a way to control and manage the interaction of light with interfaces [5], and reduce losses [4,6]. Enlarging the air core or moving to longer wavelengths are known ways to reduce roughness scattering losses. Nevertheless, in order for PBGFs to compete with classical solid-core fibres in long haul communications, the ability to measure and reduce glass surface roughness is essential.

The amorphous structure of a glass reflects the liquid state. In the same way, the glass surface inherits its roughness from the fluctuations of the liquid surface. During the fibre drawing process, the vitreous liquid goes through a glass transition in which the surface capillary waves (SCWs) that are thermally excited in the liquid state are suddenly frozen $[3,7,8]$. According to this scenario, the roughness statistics of a glass surface are therefore the same as those of a snapshot of the liquid surface taken at the glass transition temperature $T_{g}$.

This fundamental thermodynamical process imposes the ultimate limit to roughness in glasses, and in HC-PBGFs in particular. The resulting roughness is extremly low. It is controlled by the competition between the thermal noise at the glass transition $k_{B} T_{G}$ (where $k_{B}$ is the Boltzmann constant) and the surface tension $\gamma$. Typical values of the RMS roughness amount to $\sigma \approx \sqrt{k_{B} T_{G} / \gamma} \approx 0.4 \mathrm{~nm}$ for amorphous silica. This global estimate actually results from the superposition of surface capillary wave modes with spatial wavelengths that may extend from the nanometric to the millimetric range [8].

Pushed close to their instrumental limits, AFM microscopes have proven their ability to measure sub-nanometer roughness on flat glass surfaces, and to obtain roughness density spectra

\#223129 - \$15.00 USD Received 15 Sep 2014; revised 7 Nov 2014; accepted 10 Nov 2014; published 19 Nov 2014

(C) 2014 OSA 1 December $2014 \mid$ Vol. 22, No. 24 | DOI:10.1364/OE.22.029554| OPTICS EXPRESS 29555 
which are well described by theory [8]. In HC-PBGF, however, such measurements are much more difficult to perform, since it is extremely difficult to access the hollow core, typically 1020 micrometers in diameter, with an AFM. Two techniques have been developed in order to obtain AFM measurements of the inner core wall: Crushing the fibre and identifying a suitable sample among the fragments is a possibility [3]; Another possibility is to bend the fibre and polish until a sufficient portion of the inner core is exposed [9]. In both cases, and especially when polishing the fibre, contamination of the surface by other fragments, dust or lubricants is likely to affect the measurements, and stringent sample cleaning and preparation procedures are required in order to study the surface with the necessary sub-nm precision. Moreover, mechanical access to the surface over long distances remains extremely difficult with an AFM tip. The lateral and vertical resolution of the best AFMs is unrivalled, but their spatial range is relatively limited. Even if large flat portions of the sample were accessible, AFM scans with sufficient vertical resolution would be difficult to obtain over length scales at hundreds of micrometers as needed to characterize the important contribution of short spatial frequencies to the surface roughness. Surface roughness characterization at low spatial frequency is however of strong relevance for the development of low-loss HC-PBGFs. Indeed, it has been recently shown [4] that the scattering loss strongly depends on the low-frequency part of the roughness spectrum. About $50 \%$ of the total loss could be due to frequencies below $0.25 \mu^{-1}$. In this context, optical profilometry, naturally suited to the lower part of the spatial frequency interval of interest for HC-PBGFs could thus provide a complementary tool for surface roughness characterization.

Compared to AFM, optical characterization techniques offer much lower lateral resolution, limited by diffraction to approximately the wavelength of light $\lambda$ in classical microscopes. In the vertical direction, however, their precision can be as good (or even better) as that of AFM microscopes, which are themselves based on optical detection systems. Specular reflection spectroscopy has for instance recently been successfully used to probe the dynamics of surface fluctuations induced by thermal capillary waves in complex fluids $[10,11,12]$. In the context of HC-PBGF, where short scale roughness is already well characterized, and where long spatial frequencies are crucial, optical techniques offer the advantage of being contactless and non-invasive. Here, we show that a polarization-modulated differential profilometer allows the measurement of roughness of the inner surface of hollow silica capillary tubes (fibres) over hundreds of micrometers, with a picometer-range vertical precision. Moreover, the use of oil immersion optics can eliminate unwanted reflections off the outer surface of the fibres, allowing the study of the inner structure of the fibre without having to open it. This instrument, which allows long-distance scans with picometer vertical precision inside pristine fibre samples, avoiding any contamination introduced by the environment of sample handling and preparation, should prove extremely valuable in the accurate characterization of the roughness of hollow fibres.

\section{Experimental set-up: oil-immersion differential profilometry}

Differential polarization interferometers use a laser beam which is split by a Wollaston prism into two arms carrying different polarizations. After reflection of the two laser spots corresponding to each arm, separated by a distance $d$ on the surface of the sample, their interference yields a signal proportional to the optical path difference between the two beams, and therefore to the height difference $D(x)=h(x+d / 2)-h(x-d / 2)$ between them (where $x$ is the horizontal coordinate along the surface and $h(x)$ the height profile). $D(x)$ is measured while moving the sample along the $x$ direction, and the profile of the sample can then be obtained by integrating $D(x)$.

Here, we use the system described in Fig. 1, inspired by Gleyzes [13], in which a polarization modulation is introduced in order to take advantage of the high signal-to-noise ratios achieved

\#223129 - \$15.00 USD Received 15 Sep 2014; revised 7 Nov 2014; accepted 10 Nov 2014; published 19 Nov 2014

(C) 2014 OSA 1 December $2014 \mid$ Vol. 22, No. 24 | DOI:10.1364/OE.22.029554 | OPTICS EXPRESS 29556 


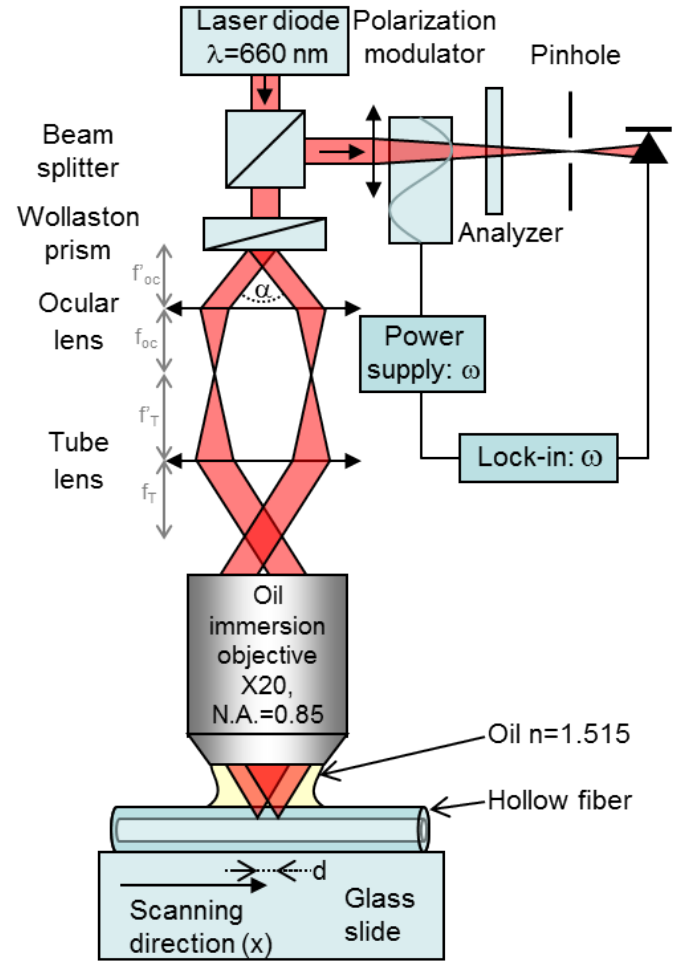

Fig. 1. Polarization-modulated differential scanning interferometer. The light of the laser diode is separated into two beams carrying orthogonal polarizations by a Wollaston prism. The immersion objective is focused on the top inner interface of the capillary tube. After reflection, these beams are recombined and sent through a photoelastic polarization modulator and sent on a laser diode for lock-in detection.

with lock-in detection. The collimated laser beam $(\lambda=660 \mathrm{~nm}, \mathrm{P}=120 \mathrm{~mW})$, polarized at $45^{\circ}$ with respect to the axes of the Wollaston prism (prism angle $\theta$ ), is again divided into two arms carrying different polarizations, separated by an angle $\alpha=2\left(n_{e}-n_{0}\right) \tan \theta \approx 5^{\prime}$ where $n_{e}$ and $n_{o}$ are the extraordinary and ordinary indices of the prism. Each beam is then focused on the investigated surface to obtain two spots separated by a distance $d=\alpha f=\alpha /\left(4 \gamma_{o} G_{o c}\right)$ where $f$ is the focal length of the microscope, $\gamma_{o}$ the magnification of the objective, and $G_{o c}$ the angular magnification of the ocular lens. After reflection on the sample, these beams are recombined in the Wollaston prism, exactly along their previous path if the Wollaston prism is in the focal plane of the microscope (see Fig. 1). The phase between the two components of the polarisation is then shifted by $\varphi=2\left(\varphi_{w}+\varphi_{s}\right)$, where $\varphi_{w}=\frac{2 \pi}{\lambda} \alpha T_{w}$ is the phase shift induced by the Wollaston prism, $T_{w}$ being the distance between the axis of the beam and the center of the Wollaston, and $\varphi_{s}=\frac{2 \pi}{\lambda} n D(x)$ is the phase difference introduced in the propagation medium (index $n$ ) if the beams hit the sample surface at heights differing by $D(x)$. After reflection on the beamsplitter, $1 / 4$ of the initial laser power is sent to the homemade photoelastic polarization modulator, in which a birefringence modulated at a frequency $F=\omega / 2 \pi=50 \mathrm{kHz}$ is created by a sinusoidal strain applied to a silica glass rod, inducing a phase modulation $\Phi(t)=\Phi_{M} \sin \omega t$. Both orthogonal polarisations are then projected on the axis of an analyzer forming an angle of $45^{\circ}$ in order to allow interference. Finally, a pinhole, conjugated with the impact spot of both 
beams on the sample, filters out stray light and parasitic reflections before measurement with a photodiode (Thorlabs PD100). The resulting signal is processed by a lock-in amplifier.

In our analysis, the surface of the glass samples are assumed to be optically homogeneous, i.e. exempt of local refractive index variations, and the reflection coefficients, $r$, are assumed identical for both beams. The intensity on the detector can thus be written as:

$$
\begin{aligned}
I(t) & =r E_{0}^{2}[1+\cos \varphi \cos \Phi(t)-\sin \varphi \sin \Phi(t)] \\
& \approx r E_{0}^{2}\left[1+J_{0}\left(\Phi_{M}\right) \cos \varphi-2 J_{1}\left(\Phi_{M}\right) \sin \varphi \sin \omega t+2 J_{2}\left(\Phi_{M}\right) \cos \varphi \cos 2 \omega t+\ldots\right]
\end{aligned}
$$

where $J_{n}$ are Bessel functions of the first kind. The third term, at the angular frequency $\omega$, is isolated by the lock-in to obtain a signal proportional to $\sin \varphi$ thus to $\varphi_{s}$ and $D(x)$. The value of the proportionality factor, which is necessary in order to quantitatively measure $\mathrm{D}(\mathrm{x})$, is easily obtained by translating the Wollaston prism to change $\varphi_{w}$ and measuring the amplitude signal for positions corresponding to $\sin \varphi=\sin 2\left(\varphi_{w}+\varphi_{s}\right)$ equal to 1 or -1 .

If we assume no overlap between the two spots, this measurement therefore delivers the height difference $D(x)$ between the two spots; the case of overlapping Gaussian laser spots is slightly more complex and will be described later.

In order to be able to measure inside hollow glass fibres, we use an oil immersion objective (Olympus X20, N.A. $=0.85$ ). A section of the fibre is fixed on a glass slide and immersed in a silica index matching oil. The outer interface of the fibre is then optically invisible, and approximately $4 \%$ of the incident light is reflected off the first glass/air interface, i.e. the upper inner surface of the glass fibre.

An important question is whether a portion of the transmitted beam can be collected after multiple reflections inside the hollow cylinder, as this might affect the measurement. Since we use a pinhole to filter out non-confocal light, light can only be collected if it emerges from the illuminated point, which has a radius $r=1.22 \lambda /(2 N . A$. $)=500 \mathrm{~nm}$. This is only possible for beams lying in a plane perpendicular to the axis of the fibre, so the problem is actually that of a $2 \mathrm{D}$ reflection at a circular surface. If a beam enters the circle at an incidence angle $i$, its reflections inside will all form the same angle $i$. After $k$ reflections, the beam impinges on the inner surface of the cylinder at an azimuthal position given by the angle $k .(\pi-2 . i)$, with relative intensities given by $\left[R_{s}(i)\right]^{k}$ and $\left[R_{p}(i)\right]^{k}$, where $R_{s}(i)$ and $R_{p}(i)$ are the Fresnel coefficients for the $s$ and $p$ polarisations, respectively. These beams can only be collected by the confocal microscope if they emerge, after $k$ reflections, at the illuminated point (radius $r$ ). We have verified numerically that, for a fibre with an internal diameter of $200 \mu \mathrm{m}$, the collected intensity for both polarizations is lower than the precision of our calculations, and is therefore negligible. This holds true down to fibre radii of the order of the wavelength, i.e. a few hundreds of nanometers, at which scale this geometrical approach is no longer valid.

The sample is then scanned relative to the beams using a motorized stage (PI M-126-2S1), to obtain a line scan $D(x)$. The differential interferometer is mostly insensitive to unwanted vertical movements, but pitch or yaw angular tilts are crucial, since a sample rotation of e.g. $\rho=10^{-6} \mathrm{rad}$. results in an apparent differential displacement $\rho d=3 \mathrm{pm}$. However, most good quality stages can provide this kind of precision if the scanning direction is kept constant.

An important characteristic of this type of interferometer is that, since the angle $\alpha$ is small $\left(5^{\prime}\right)$, both beams follow very similar paths and are affected in similar ways by air-flow and vibrations. This makes the instrument relatively immune to these sources of noise.

The ultimate noise limit, which is driven by photon and detector noise, is of the order of $10^{-14} \mathrm{~m}$ for a lock-in integration time of $\tau=100 \mathrm{~ms}$, but it is in practice limited to higher values by vibrations and thermal drifts. In order to estimate this noise experimentally, we have carried out repeated measurements on a fixed region for different types of samples, measuring the fluctuations of the signal. Figure 2 depicts the results obtained. The noise level is plotted as a

\#223129 - \$15.00 USD Received 15 Sep 2014; revised 7 Nov 2014; accepted 10 Nov 2014; published 19 Nov 2014 (C) 2014 OSA 1 December $2014 \mid$ Vol. 22, No. 24 | DOI:10.1364/OE.22.029554| OPTICS EXPRESS 29558 


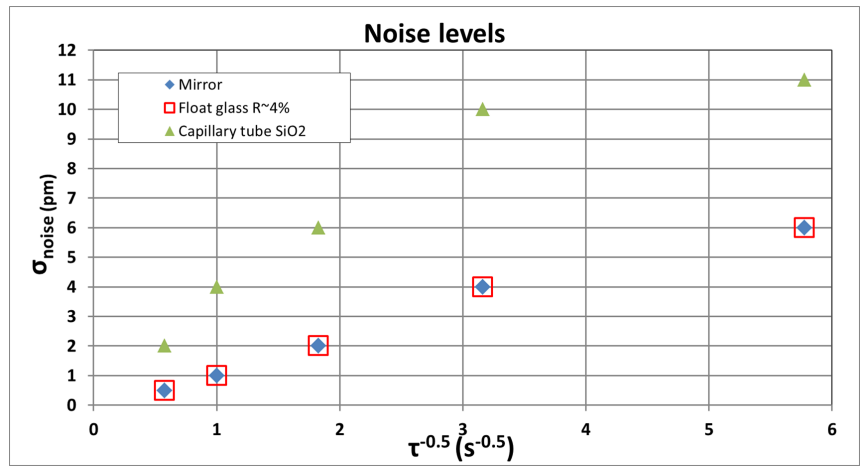

Fig. 2. Current performances Noise levels measured on different kinds of samples. $\tau$ is the time constant of the lock-in amplifier.

function of one over the square root of the time constant of the lock-in amplifier. As in most lock-in detection systems, drifts also appear in the amplitude signal. In all the experiments presented in this paper, we adopted the conservative assumption that these drifts were linear, substracting a linear fit over the raw measurements.

On a perfectly reflecting surface such as a mirror, the noise level reaches the practical noise limit, which is around $4 p m$ for $\tau=100 \mathrm{~ms}$. Since glasses only reflect about $4 \%$ of the incoming light, the available number of photons is notably decreased and the signal to noise ratio is therefore reduced. Note that by increasing the power of the laser diode until we obtain a reflected signal which has the same intensity as on a mirror, we find the same noise levels. However, immersion measurements on capillary fibres lead to higher noise levels which might be caused by movements in the oil, and by the very low depth of field of immersion objectives.

Within the time constant range used to measure differential profiles (a few $m s$ ), the noise levels are around six to ten picometers. This allows precise measurements of the glass roughness. Moreover, we note that, even though the lateral resolution of the differential profilometer is as expected lower than that of an atomic force microscope, the vertical noise level of immersion measurements is at least three times lower than that of an AFM that typically amounts to $30 \mathrm{pm}$ for the low noise commercial instrument used in the present study [14].

\section{Response function}

In the following sections we present results obtained on two different kinds of samples. Test silica samples that exhibit a deterministic step height profile of nanometric amplitude will first be examined. Hollow silica tubes characterized by a sub-nanometric random roughness will then be studied. In both cases, results will be quantitatively compared either with independent experimental results (AFM measurements on the silica steps) or with theoretical expectations (frozen capillary waves for the inner roughness of capillary tubes). While the quantitative analysis of a deterministic height profile naturally resorts to the real space, the characterization of a random roughness will be more practical in the Fourier space.

Before coming to the experimental results we give here more details on the experimental response function and its effects on the measurements. The two cases discussed above are presented and both the real space and the Fourier space representations of the response functions are discussed.

As presented in the above section, differential profilometry gives access to a local phase difference $\varphi_{s}(x)=(2 \pi n / \lambda) D(x)$ on the sample surface due to the difference of optical path 
between the two reflected beams. If the distance $d$ separating the two beams is significantly larger than the beam waist $w, d>>w$ we expect that the measured signal directly gives the height difference between the two points of reflection:

$$
D(x) \approx h(x+d / 2)-h(x+d / 2) \approx d \cdot h^{\prime}(x)
$$

In practice however, measurements are performed with a separation between the two beams of the same order of magnitude as the beam waist, $d / w \approx 1-3$ and the two beams can partially overlap. For small values of $d$, the response of the profilometer is thus smeared out, essentially due to the diffraction limit which sets the diameter of the spots.

\subsection{Step geometry-Real space response function}

Let us first consider the case of a step profile of height $M$. The height difference $D$ measured by the profilometer can be written as:

$$
D(x)=\frac{\lambda}{4 \pi} \arcsin \left[A(x) \sin \left(\frac{4 \pi M}{\lambda}\right)\right] .
$$

In particular the maximum of the signal $D_{\max }=D(0)$ is corrected by a factor $A(0)$ which is equal to unity when the two beams are fully separated and takes lower and lower values when the beams overlap. A Gaussian hypothesis for the shape of the beams leads to:

$$
A(x)=\frac{1}{2}\left[\operatorname{erf}\left(\frac{x+d / 2}{w}\right)-\operatorname{erf}\left(\frac{x-d / 2}{w}\right)\right] .
$$

For steps of small height $M \ll \lambda$, linearization gives:

$$
D(x) \approx M A(x)=\frac{M}{2}\left[\operatorname{erf}\left(\frac{x+d / 2}{w}\right)-\operatorname{erf}\left(\frac{x-d / 2}{w}\right)\right] .
$$

Here, $A(x)$ is thus nothing but the linear response function of the experimental set-up to a unit step. The main effect of the response function in this experimental configuration is an underestimate of the step height $M$. The maximum of the signal $D_{\max }=D(0)$ is corrected by a factor $A(0)$ which is equal to unity when the two beams are fully separated and takes lower and lower values when the beams overlap.

\subsection{Random roughness-Fourier space response function}

Following Eq. (4) the linear response function of the experimental set-up to a height Dirac is:

$$
R(x)=A^{\prime}(x)=G(x+d / 2)-G(x-d / 2),
$$

where $G$ is the normalized Gaussian function of width $w$ :

$$
G(x)=\frac{1}{w \sqrt{\pi}} \exp \left[-\left(\frac{x}{w}\right)^{2}\right] .
$$

The Fourier transform of the response function thus writes:

$$
\widetilde{R}(f)=2 i e^{-\pi^{2} f^{2} w^{2}} \sin (\pi f d),
$$

where the Fourier transform is defined as:

$$
\widetilde{g}(f)=\int_{-\infty}^{\infty} g(x) e^{2 i \pi f x} d x .
$$

\#223129 - \$15.00 USD Received 15 Sep 2014; revised 7 Nov 2014; accepted 10 Nov 2014; published 19 Nov 2014 (C) 2014 OSA 1 December $2014 \mid$ Vol. 22, No. 24 | DOI:10.1364/OE.22.029554 | OPTICS EXPRESS 29560 


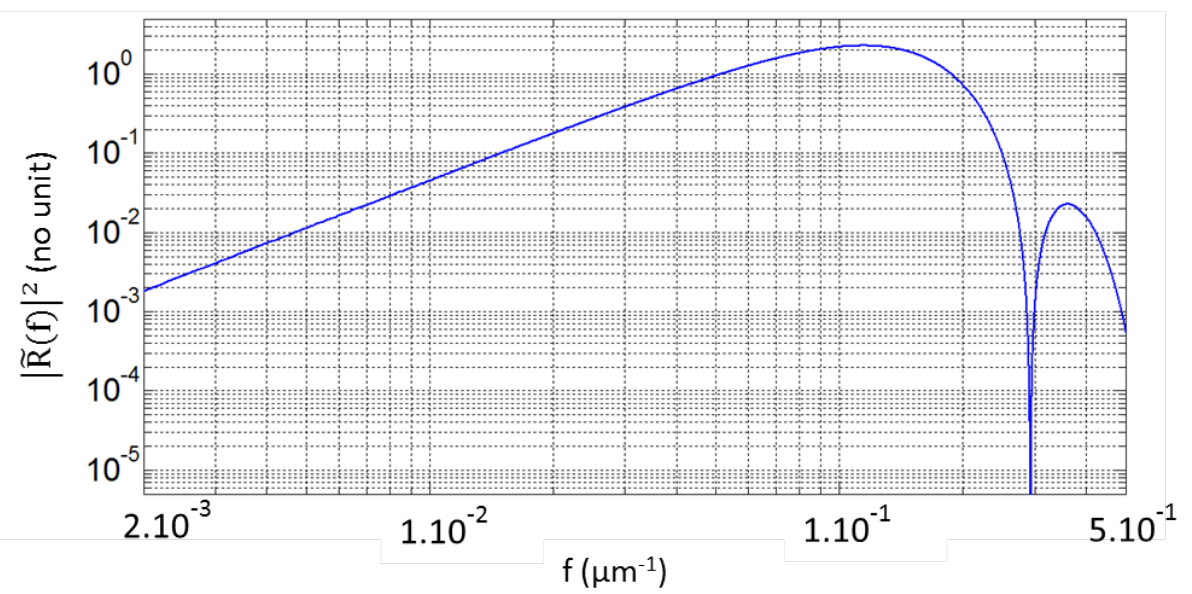

Fig. 3. Response function $|\widetilde{R}(f)|^{2}$ for a beam waist $w=1.3 \mu \mathrm{m}$ and a beam separation $d=3.4 \mu \mathrm{m}$. At low frequency, the profilometer acts as a differential operator and and we get the parabolic behavior $|\widetilde{R}(f)|^{2} \propto f^{2}$. In addition to the diffraction effect at high frequency, the zero induced by the differential operator is clearly visible at frequency $f_{d}=1 / d=$ $0.29 \mu^{-1}$, and can provide an experimental determination of $d$.

At low frequencies, $f<<1 / d$, one retrieves the Fourier transform of the differential operator $\widetilde{R}(f) \approx 2 i \pi f d$. Note here that the attenuation factor $A(0)$ discussed in the previous subsection for the measurements of the steps results from the low pass filter and not from a mechanism that would attenuate all frequencies.

The differential nature of the measurement set-up has another important feature. In Fig. 3 we have plotted the square modulus of the Fourier transform of the response function, $|\widetilde{R}(f)|^{2}$, using the beam waist value $w=1.3 \mu \mathrm{m}$ (determined from experimental measurements). Larger values of $w$ (i.e. larger laser spots in case of e.g. imperfect focusing) will decrease the sensitivity to high spatial frequencies. As can be seen in Fig. 3, $|\widetilde{R}(f)|^{2}$ has a sharp minimum corresponding to the spatial frequency $f_{d}=1 / d$, the frequency to which our measurement system is entirely blind. This frequency therefore provides a way to measure accurately the beam separation. We get $f_{d}=0.29 \mu \mathrm{m}^{-1}$ for immersion measurements, the corresponding value $d=3.4 \mu \mathrm{m}$ was used throughout our analysis.

If we denote $h(x)$ and $\widetilde{h}(f)$ the random height profile under measurement and its Fourier transform respectively, we get for the differential signal:

$$
\widetilde{D}(f)=\widetilde{R}(f) \cdot \widetilde{h}(f)=2 i e^{-\pi^{2} f^{2} w^{2}} \sin (\pi f d) \widetilde{h}(f)
$$

While the integration of the differential measurement would require a simple division by the response function in the Fourier space, the blindness of the set-up at $f_{d}=1 / d$ makes this operation numerically hazardous. Since it involves a division by zero, integration in the Fourier space is thus only permitted in a frequency range strictly below $f_{d}=1 / d$ [13]. While this operation is possible it obviously reduces the range of accessible frequencies.

Another procedure consists in accounting for the response function and trying to directly analyze the bare differential signal. Let us consider a random height profile $h(x)$. Such a random signal can be characterized by its statistical distribution and by its spatial correlation $C(x)$. The 
power spectral density (PSD) of the differential signal will thus simply writes:

$$
\begin{aligned}
|\widetilde{D}(f)|^{2} & =|\widetilde{R}(f)|^{2} \widetilde{C}(f) \\
& =4|\sin (\pi f d)|^{2} e^{-2 \pi^{2} f^{2} w^{2}}|\widetilde{h}(f)|^{2}
\end{aligned}
$$

where we have $\widetilde{C}(f)=|\widetilde{h}(f)|^{2}$.

For the sake of simplicity let us distinguish two different cases: short-range gaussian correlations and long-range correlation. In the first case the width of the distribution is given by the RMS roughness $\sigma$ and the height-height correlation function $C(x)=\sigma^{2} \exp \left(-x^{2} / \tau^{2}\right)$ is additionally characterized by the correlation length $\tau$. We thus get:

$$
\begin{aligned}
\widetilde{C}(f) & =\sqrt{\pi} \sigma^{2} \tau e^{-\pi^{2} f^{2} \tau^{2}} \\
|\widetilde{D}(f)|^{2} & =4 \sqrt{\pi} \sigma^{2} \tau|\sin (\pi f d)|^{2} e^{-\pi^{2} f^{2}\left(2 w^{2}+\tau^{2}\right)}
\end{aligned}
$$

Here the RMS roughness level simply gives a multiplicative factor while the gaussian correlation induces a low pass filter only measurable if the correlation length $\tau$ is significantly larger than the beam separation $d$. In particular at low frequency we expect in such a case to get a parabolic dependence of the PSD to the frequency: $|\widetilde{D}(f)|^{2} \approx 4 \pi^{5 / 2} \sigma^{2} \tau d^{2} f^{2}$.

Let us now consider a second case of roughness that exhibits long range correlations. Glass surfaces obtained by a quench from liquid state show an ultra-low (sub-nanometric) roughness. It appears that this roughness can be quantitatively described as a superposition of frozen capillary waves $[3,7,8]$. In the same way as the bulk structure of glass can be regarded as a snapshot of the liquid at the temperature of glass transition $T_{G}$, glass surface is here regarded as a snapshot of the thermal fluctuations of the liquid interface at $T_{G}$. If we call $h_{S C W}$ the height fluctuations induced by surface capillary waves, the power spectral density expected over $1 D$ profiles can be expresssed as:

$$
\left|\widetilde{h}_{S C W}(f)\right|^{2}=\frac{k_{b} T_{G}}{2 \pi \gamma f},
$$

where $k_{B}$ is the Boltzmann constant, $T_{G}$ the glass transition and $\gamma$ the surface tension. Here $k_{b} T_{G} / \gamma$ has the dimension of the square of a length and its square root gives a good estimate of the width of the interface. After accounting for the response function we get for the PSD of the differential signal:

$$
\begin{aligned}
\left|\widetilde{D}_{S C W}(f)\right|^{2} & =4 e^{-2 \pi^{2} f^{2} w^{2}}|\sin (\pi f d)|^{2} \frac{k_{b} T_{G}}{2 \pi \gamma f}, \\
& \approx 2 \pi d^{2} \frac{k_{b} T_{G}}{\gamma} f \text { if } f \ll \frac{1}{d} .
\end{aligned}
$$

Here we see that while a short range correlated roughness does not alter the low freqency behavior of the differential signal, the situation is very different in the case of the long-range correlated roughness induced by frozen capillary waves. The PSD shows now a linear frequency behavior.

In the following sections we make use of Eq. (5) and (16) to fit the results obtained on a deterministic silica step sample and on the inner roughness of hollow capillary silica tubes, respectively.

\section{Tests on textured silica surfaces}

In order to investigate the performance of our setup on representative test samples we performed measurements of textured silica wafers. We used $100 \mu \mathrm{m}$ thick amorphous silica wafers (Corning 7980 Fused silica UV grade), with a standard surface finish (Optically polished both sides $<$

\#223129 - \$15.00 USD Received 15 Sep 2014; revised 7 Nov 2014; accepted 10 Nov 2014; published 19 Nov 2014

(C) 2014 OSA 1 December 2014 | Vol. 22, No. 24 | DOI:10.1364/OE.22.029554| OPTICS EXPRESS 29562 

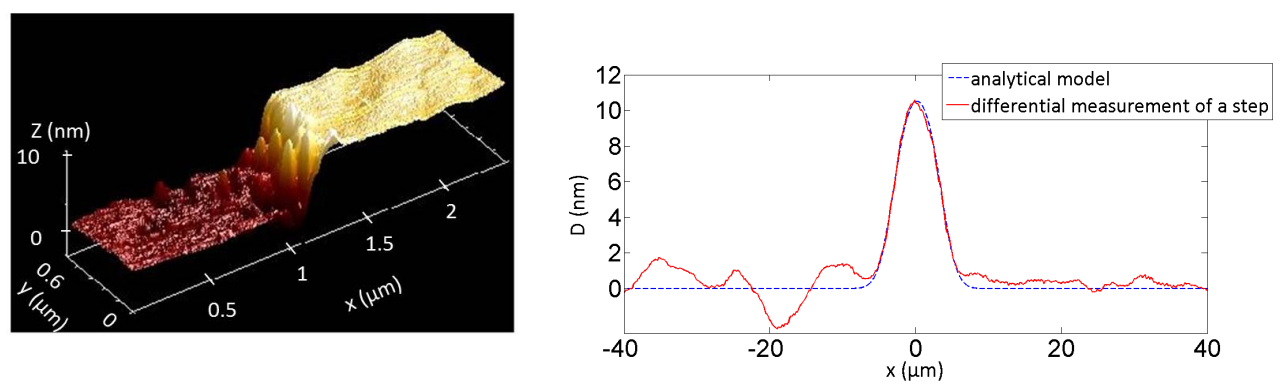

Fig. 4. Tests on silica steps obtained in air by RIE etching (exposure time 15s). Left: AFM image (estimated step height $11.7 \mathrm{~nm}$ ) Right: Differential profilomety measurement obtained at the glass/air interface on the same step (estimated step height $11.1 \mathrm{~nm}$ after fitting with analytical expression (5), using $d=6.5 \mu \mathrm{m}$ and $w=2.1 \mu \mathrm{m}$ ). The displacement step size used for the measurement was $0.18 \mu \mathrm{m}$.

20/10 scratch/dig). Steps of known height were fabricated in these samples by use of Reactive Ion Etching (RIE) with processing time ranging from $8 \mathrm{~s}$ to $120 \mathrm{~s}$. The profiles of the steps were measured by AFM (see Fig. 4). The height of the steps was found to range from $4.5 \mathrm{~nm}$ to 85 $\mathrm{nm}$. As can be seen from the AFM image, the steps are extremely sharp with a typical transition width estimated to be about $70 \mathrm{~nm}$, i.e. far below the resolution of optical profilometry.

After AFM measurements, the test samples were measured by differential profilometry i) in the conventional air-reflection configuration, and ii) in the new immersion configuration.

These test samples are planar and the height of the steps is obviously significantly larger than the sub-nanometric roughness of optical fibres. Still, the use of thin samples made of the same material (silica) as the fibres enables us to test our instrument in conditions of low sample reflectivity, and in the case of oil-immersion measurements, after transmission in the bulk material that are similar to those met in the case of fibres. The sharpness of the steps gives us the possibility to use equation (5) derived in the previous section to fit the experimental measurements. An example of a differential profile is given in Fig. 4 for a $10 \mathrm{~nm}$ high step. One obtains an excellent description of the profile with the predicted analytical expression. The parameters $d$ and $w$ were obtained by measuring $f_{d}$ and through the fit on the experimental measurements respectively, yielding $d=6.5 \mu \mathrm{m}$ and $w=2.1 \mu \mathrm{m}$ in air and $d=3.4 \mu \mathrm{m}$ and $w=1.3 \mu \mathrm{m}$ in oil immersion. AFM and air and immersion measurements were performed on a set of 5 samples textured with steps obtained by RIE exposure of respectively, 8s, 15s, 30s, $60 \mathrm{~s}$, and 120s. For each sample 8 AFM images, 8 differential profiles in air, and 8 profiles in immersion were performed. The results are summarized in Table 1.

For AFM measurements, $10 \mu \mathrm{m} \times 5 \mu \mathrm{m}$ images were acquired in contact mode on an Icon (Brücker) AFM with a tip stiffness of $0.2 \mathrm{~N} . \mathrm{m}^{-1}$ and a scan rate of $1 \mathrm{~Hz}$. Planar fits (or flattening fits i.e. line by line along the fast scan direction) are performed on either the lower or the upper area at some distance from the step. After fitting the height histogram, two well defined peaks are identified. The distance between the two peaks is computed.

Table 1 summarizes the mean value and the standard deviation obtained over the differen$\mathrm{t}$ realizations for AFM, air and immersion differential profilometry. The different techniques give very consistent estimates. RIE etching times are roughly proportional to the actual etching depth as confirmed, with a much better accuracy, by AFM or optical profilometry. Note that even if measurements are performed on deterministic samples, i.e. steps, the results still have to be interpreted within a statistical framework. The main reason is that the RIE process is not expected to produce a perfect homogeneous step. Two main effects can be invoked here. First, the 
Table 1. Height of silica steps made by RIE, as measured by AFM and by differential profilometry in air and in immersion. The heights obtained by optical profilometry correspond to the parameters $\mathrm{M}$ providing the best fits to the experimental data using expression (5).

\begin{tabular}{|c|c|c|c|}
\hline RIE exposure time & AFM & Air & Immersion \\
\hline $8 \mathrm{~s}$ & $4.7 \pm 0.6 \mathrm{~nm}$ & $4.6 \pm 0.4 \mathrm{~nm}$ & $4.3 \pm 0.3 \mathrm{~nm}$ \\
\hline $15 \mathrm{~s}$ & $12.2 \pm 1.0 \mathrm{~nm}$ & $11.2 \pm 1.0 \mathrm{~nm}$ & $10.3 \pm 0.8 \mathrm{~nm}$ \\
\hline $30 \mathrm{~s}$ & $20.9 \pm 1.7 \mathrm{~nm}$ & $22.1 \pm 1.5 \mathrm{~nm}$ & $19.1 \pm 1.3 \mathrm{~nm}$ \\
\hline $60 \mathrm{~s}$ & $45.5 \pm 2.5 \mathrm{~nm}$ & $48.3 \pm 1.8 \mathrm{~nm}$ & $46.8 \pm 3.7 \mathrm{~nm}$ \\
\hline $120 \mathrm{~s}$ & $84.3 \pm 2.5 \mathrm{~nm}$ & $79.6 \pm 4.0 \mathrm{~nm}$ & $86.8 \pm 2.4 \mathrm{~nm}$ \\
\hline
\end{tabular}

intensity of RIE etching may spatially vary (over large length scales) and owing to the different scales of lateral resolutions of the two instruments, AFM and differential profilometry can not probe the very same area. The intrinsic roughness of the silica sample and that induced by the etching process (altogether a RMS roughnes of $2 \mathrm{~nm}$ was estimated on the samples) induces a significant statistical uncertainty in the estimate of the step height. The results presented above with the different experimental techniques are therefore extremely consistent, and provide a cross validation of the AFM and differential profilometry.

\section{Measurements on inner surfaces of hollow glass fibres}

Direct measurements on the inner surfaces of hollow glass fibres have been performed using immersion differential profilometry. Two different samples have been studied. A hollow pure silica fibre of outer diameter (OD) $1350 \mu \mathrm{m}$ and wall thickness (WT) $88 \mu \mathrm{m}$ and another capillary tube of OD $950 \mu \mathrm{m}$ and WT $75 \mu \mathrm{m}$ made of alumina-doped silica. The first sample was produced by drawing a Suprasil F300 silica tube from Heraeus using a conventional fibre draw tower. The second sample was prepared using a conventional solution doping technique and then drawn in the same way as the first one, aside from the slightly different diameter. 11 independent profiles were measured on each of the two samples.

During the drawing process the samples experience heating at a temperature of about $2000^{\circ} \mathrm{C}$ i.e. far above the glass transition temperature $T_{G}$ of the materials $\left(T_{G} \approx 1250^{\circ} \mathrm{C}\right.$ for silica). After cooling below $T_{G}$, the roughness of the glass surface is therefore expected to result from the subsequent freezing of surface capillary waves $[3,7,8]$ with their amplitude controlled by the ratio $T_{G} / \gamma$ where $\gamma$ is the interface tension between the viscous glass and the surrounding atmosphere at $T_{G}$. The two samples have different compositions and different values of $T_{G}$ and $\gamma$, and are thus expected to have different thermodynamic roughnesses.

The purpose of this investigation is i) to test whether the roughness of the inner surfaces of the fibres can be quantitatively characterized in terms of surface capillary waves in the accessible spatial frequency range, and ii) to establish whether our technique is able to resolve the difference between the two materials. In the present case the composition change (presence of alumina in one of the two samples) affects these parameters and is a potential cause of roughness contrast between the two fibres.

In Fig. 5 we show a differential profile obtained on the inner surface of a hollow silica fibre over $200 \mu \mathrm{m}$. The height fluctuations appear to be extremely low, in the range of a few hundred$\mathrm{s}$ of picometers. Such an ultra-low level of surface roughness is compatible with expectations from a frozen capillary wave scenario. A more quantitative confirmation requires characterization of the Fourier spectrum of the height profile to test whether it is consistent with the

\#223129 - \$15.00 USD Received 15 Sep 2014; revised 7 Nov 2014; accepted 10 Nov 2014; published 19 Nov 2014 (C) 2014 OSA 1 December 2014 | Vol. 22, No. 24 | DOI:10.1364/OE.22.029554 | OPTICS EXPRESS 29564 


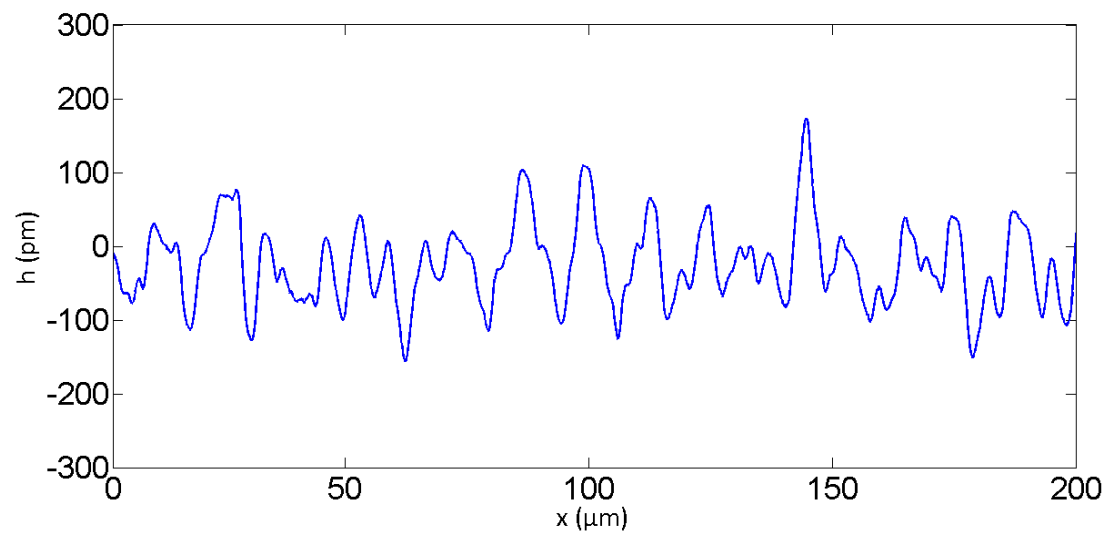

Fig. 5. Differential profile obtained on the inner surface of a silica capillary tube over a length of $200 \mu \mathrm{m}$. Height fluctuations are extremely low, in the range of a few hundreds of picometers.

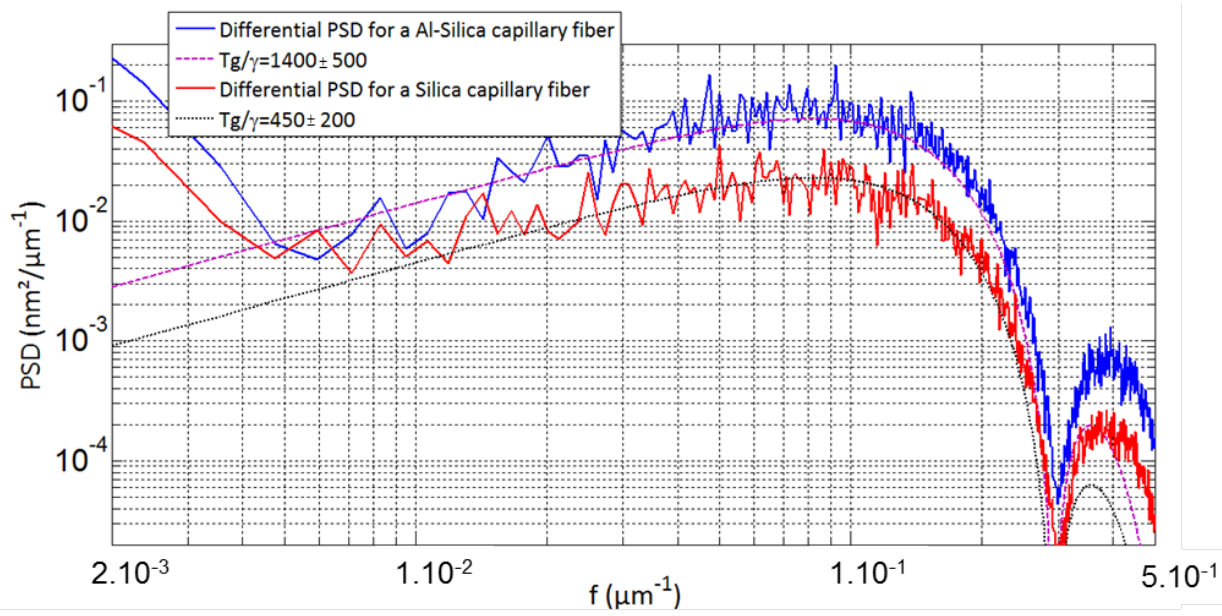

Fig. 6. Differential profilometry measurements on inner surfaces of two hollow glass fibres (silica and alumina doped silica). The power spectral densities of the differential height signal are represented as continuous lines. The dashed lines are fits obtained with the analytical expression (16). The linear trend observed at low frequency is consistent with a scenario of frozen capillary waves at the glass surface.

predicted spectrum of capillary waves. In particular, as discussed above the latter is expected to show a $1 / f$ behavior (where $f$ is the spatial frequency).

Here we first show the comparison of the power spectral density of the bare differential signal $|\widetilde{D}(f)|^{2}$ with the theoretical expectation $\left|\widetilde{D}_{S C W}(f)\right|^{2}$ obtained in Eq. (16) for a spectrum of surface capillary waves accounting for the experimental response.

The average power spectral densities of the differential signal obtained for the silica fibre and the alumina-doped fibre are displayed in Fig. 6. The linear trend (see Eq. (17)) expected for frozen capillary waves is clearly visible in the range $\left[5 \cdot 10^{-3} \mu \mathrm{m}^{-1}-5 \cdot 10^{-2} \mu \mathrm{m}^{-1}\right]$. But as 


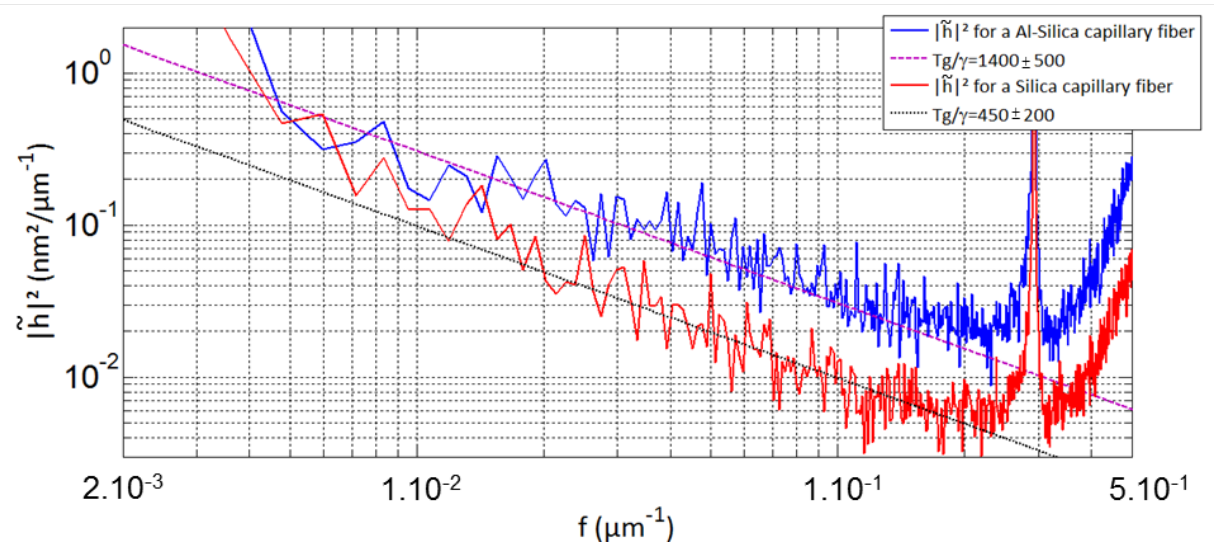

Fig. 7. Roughness measurements on inner surfaces of two hollow glass fibres (silica and alumina doped silica). The power spectral densities of the height signal are obtained after division by the response function. The dashed and dotted lines are fits obtained with the analytical expression (15). The clear $1 / f$ trend observed at low frequency is consistent with a scenario of frozen capillary waves at the glass surface.

shown in Fig. 6, beyond this linear trend, the expression (16) accounting for the experimental response function appears to describe quantitatively the experimental power spectral densities over most of the accessible range of spatial frequencies. In practice an excellent agreement is found in the range $\left[5 \cdot 10^{-3} \mu \mathrm{m}^{-1}-10^{-1} \mathrm{\mu m}^{-1}\right]$ as shown by the dashed lines in Fig. 6 . At higher frequencies the signal is filtered by diffraction. To our knowledge, this is the first time that frozen capillary waves are identified in this frequency range on glass surfaces. A larger magnification may slightly enlarge the accessible frequency range toward high frequencies albeit at the price of a reduced signal-to-noise ratio. At low frequencies (below $5 \cdot 10^{-3} \mu \mathrm{m}^{-1}$ ) the signal is dominated by contributions of the nanometric scale vertical fluctuations of the motorized stage.

In order to make the expected $1 / f$ behavior of the roughnes spectrum even more visible we have also performed a division by the response function in the frequency range $\left[2 \cdot 10^{-3} \mathrm{\mu m}^{-1}\right.$ $\left.5 \cdot 10^{-1} \mu \mathrm{m}^{-1}\right]$. As shown in Fig. 7, a very clear $1 / f$ behavior is obtained at low frequency. At higher frequency the proximity of the zero of the response function at $f_{d}=1 / d$ induces a high level of noise.

The power spectral density of the differential profiles obtained on the inner surfaces of the hollow glass fibres can thus be quantitatively accounted for by a superposition of frozen surface capillary waves. It is then possible to extract a single parameter $T_{G} / \gamma$ quantifying the amplitude of the surface capillary modes.

From the quantitative point of view, we obtain as a central value $T_{G} / \gamma=450 \pm 200$ for the silica fibre and $T_{G} / \gamma=1400 \pm 500$ for the alumina-silica fibre. The error bars have been determined after identifying lower and upper values bounding the fluctuations of the power spectral density in the range $\left[5 \cdot 10^{-3} \mu \mathrm{m}^{-1}-5 \cdot 10^{-2} \mu \mathrm{m}^{-1}\right]$.

The measurement clearly indicates a substantial difference in surface roughness for the two analyzed samples, and we speculate that the difference is a consequence of their different compositions, since both the temperature of the glass transition and the interface tension are expected to depend on composition.

Still we remark that the two values are well below the equilibrium value estimate for amorphous silica $T_{G} / \gamma \approx 5000$ obtained from the standard values for the temperature fo glass tran- 
sition $T_{G} \approx 1500 \mathrm{~K}$ and surface tension $\gamma \approx 0.3 \mathrm{~J} \cdot \mathrm{m}^{-1}$. Note that low values of $T_{G} / \gamma$ had already been reported in [3]. In this work, AFM measurements on a fragment of photonic band gap fibre led to estimates of the ratio $T_{G} / \gamma$ in the range [500-5000] with a central value $T_{G} / \gamma=1500$. A possible origin of this lower than expected surface roughness of glass fibres may lie in the drawing process. Recent experimental and numerical results suggest indeed that capillary waves may be attenuated under flowing conditions $[15,16]$.

\section{Conclusion}

We have presented an optical, non-contact method able to characterize the roughness of ultrasmooth glass surfaces with sensitivities down to $10 \mathrm{pm}$, and have validated its ability to deliver quantitative height measurements on model samples. On flat glass surfaces, the capillary wave-driven sub-nanometer roughness has been obtained in a new spatial frequency range $\left[5 \cdot 10^{-3} \mu \mathrm{m}^{-1}-10^{-1} \mu \mathrm{m}^{-1}\right]$ with a quantitative statistical charactarization of the spatial correlations. These measurements compare well to glass physics models as well as to AFM measurements [8]. Beyond its excellent sensitivity, which goes beyond the performances of most current AFM regarding height measurements, a further advantage of this method resides in its ability to obtain measurements over distances of the order of hundreds of micrometers. Using an oil immersion technique, we show that these measurements can be performed at a distant glass/air interface, either on the back side of thin glass plates or, more interestingly, inside hollow glass tubes. This immersion-based method allows the study of unopened hollow fibres, thus avoiding the necessity to mechanically open the fibre and therefore preserving the pristine cleanness of the surface, which is essential considering the extremely low roughness levels measured here. The ability to access the inner surface of glass tubes, coupled with the ability to measure a previously unexplored range of spatial frequencies at the very high sensitivity presented here is expected to be of great interest for the study of roughness and loss mechanisms in photonic bandgap fibres, providing valuable input to their understanding and optimization.

\section{Acknowledgements}

This work was supported by the EU 7th Framework Programme under grant agreement 228033 (MODE-GAP). X.B. acknowledges the support of Programme DIM OxyMORE, Ile de France. The authors thank the technology center MIMENTO, Femto ST institute, for the realization of the silica steps.

\#223129 - \$15.00 USD Received 15 Sep 2014; revised 7 Nov 2014; accepted 10 Nov 2014; published 19 Nov 2014

(C) 2014 OSA 1 December $2014 \mid$ Vol. 22, No. 24 | DOI:10.1364/OE.22.029554 | OPTICS EXPRESS 29567 